\title{
Oxidative capacity and hemolytic activity of settled dust from moisture-damaged schools
}

\author{
Kati Huttunen ${ }^{1,2,3}$ (D) | Anna J. Wlodarczyk ${ }^{2,3}$ | Jenni Tirkkonen ${ }^{1}$ | Santtu Mikkonen ${ }^{4}$ | \\ Martin Täubel $^{5}$ | Esmeralda Krop ${ }^{6}$ | José Jacobs ${ }^{6}$ | Juha Pekkanen ${ }^{5,7}$ | \\ Dick Heederik $^{6}$ | Jan-Paul Zock ${ }^{8,9,10}$ | Anne Hyvärinen ${ }^{5}$ | Maija-Riitta Hirvonen ${ }^{1,3}$ | \\ Rachel Adams $^{11}$ | Tim Jones ${ }^{12}$ | Ralf Zimmermann ${ }^{3,13}$ | Kelly BéruBée, ${ }^{2,3,14}$ \\ ${ }^{1}$ Department of Environmental and Biological Sciences, University of Eastern Finland, Kuopio, Finland \\ ${ }^{2}$ School of Biosciences, Cardiff University, Cardiff, UK \\ ${ }^{3}$ Helmholtz Virtual Institute of Complex Molecular Systems in Environmental Health (HICE), Neuherberg, Germany \\ ${ }^{4}$ Department of Applied Physics, University of Eastern Finland, Kuopio, Finland \\ ${ }^{5}$ Environmental Health Unit, Department of Health Security, National Institute for Health and Welfare, Kuopio, Finland \\ ${ }^{6}$ Division Environmental Epidemiology, Institute for Risk Assessment Sciences, Utrecht University, Utrecht, The Netherlands \\ ${ }^{7}$ Department of Public Health, University of Helsinki, Helsinki, Finland \\ ${ }^{8}$ ISGlobal, Centre for Research in Environmental Epidemiology (CREAL), Barcelona, Spain \\ ${ }^{9}$ Universitat Pompeu Fabra (UPF), Barcelona, Spain \\ ${ }^{10}$ CIBER Epidemiología y Salud Pública (CIBERESP), Madrid, Spain \\ ${ }^{11}$ Cardiff School of Health Sciences, Cardiff Metropolitan University, Cardiff, UK \\ ${ }^{12}$ School of Earth \& Ocean Sciences, Cardiff University, Cardiff, UK \\ ${ }^{13}$ Joint Mass Spectrometry Centre, Institute of Chemistry, Analytical Chemistry, University of Rostock, Rostock, Germany \\ ${ }^{14}$ Cardiff Institute for Tissue Engineering \& Repair (CITER), Cardiff University, Cardiff, Wales, UK
}

\section{Correspondence}

Kati Huttunen, Department of

Environmental and Biological Sciences,

University of Eastern Finland (UEF), Kuopio,

Finland.

Email:kati.huttunen@uef.fi

Funding information

The Helmholtz Virtual Institute of Complex Molecular Systems in Environmental Health (HICE); Maj ja Tor Nesslingin Säätiö; Olvi Foundation; Seventh Framework Programme, Grant/Award Number: 211488; The Research Foundation of the Pulmonary Diseases and The Cardiff Institute for Tissue Engineering \& Repair (CITER); Väinö and Laina Kivi Foundation; The Academy of Finland Centre of Excellence, Grant/Award Number: 272041; European Respiratory Society, Grant/Award Number: LTRF 2013 - 1505; European Commission; Generalitat de Catalunya

\begin{abstract}
Exposure to moisture-damaged indoor environments is associated with adverse respiratory health effects, but responsible factors remain unidentified. In order to explore possible mechanisms behind these effects, the oxidative capacity and hemolytic activity of settled dust samples $(n=25)$ collected from moisture-damaged and nondamaged schools in Spain, the Netherlands, and Finland were evaluated and matched against the microbial content of the sample. Oxidative capacity was determined with plasmid scission assay and hemolytic activity by assessing the damage to isolated human red blood cells. The microbial content of the samples was measured with quantitative PCR assays for selected microbial groups and by analyzing the cell wall markers ergosterol, muramic acid, endotoxins, and glucans. The moisture observations in the schools were associated with some of the microbial components in the dust, and microbial determinants grouped together increased the oxidative capacity. Oxidative capacity was also affected by particle concentration and country of origin. Two out of 14 studied dust samples from moisture-damaged schools demonstrated some hemolytic activity. The results indicate that the microbial component
\end{abstract}


connected with moisture damage is associated with increased oxidative stress and that hemolysis should be studied further as one possible mechanism contributing to the adverse health effects of moisture-damaged buildings.

KEYWORDS

hemolysis, microbes, moisture, oxidative capacity, reactive oxygen species, settled dust

\section{1 | INTRODUCTION}

Particulate matter (PM) in the indoor environment may contain several toxic or bioactive components such as metals, polycyclic aromatic hydrocarbons (PAH), pollen, microbes, and their metabolites. Increased health risks have been associated particularly with particles originating from combustion processes and microbial growth enabled by excess moisture within the building structures. ${ }^{1}$ The toxicity of PM is affected by the size, components, and emission source of the particles; thus, the hazard resulting from exposure is difficult to predict. This has inspired the development of cellular biosensors for assessing the extent and type of toxicity associated with exposure. $^{2}$

Immunotoxicological activity of indoor air PM has been studied most often with immunocytes ${ }^{3-6}$ or lung epithelial cells. ${ }^{7,8} \mathrm{~A}$ recent study showed an increase in the immunotoxicological activity of dust from school buildings with higher microbial load, although not consistently associated with moisture-damage status of the building or symptoms of the occupants. ${ }^{9}$ The failure to reliably identify moisture-damaged indoor environments with bioassays may indicate that the model system simply does not reflect the health responses of occupants accurately enough, but it could also be biased, for example, by other bioactive materials in the sample, the sampling method, or measured toxicological endpoints.

The choice of biomarkers of exposure is not straightforward, as the exposure to moisture-damaged indoor environments could cause adverse health effects via several different mechanisms. The strongest evidence is for chronic inflammation and damage to respiratory tissue caused by exposure to fungi and bacteria, although causality remains an issue of debate. Several types of microbial exposure have been connected with indoor air-related symptoms. ${ }^{10-12}$ Importantly, the effect of co-exposure to microbes and their components has been found to be synergistic, ${ }^{13,14}$ which supports studying the effects of real-life combinations of exposure agents instead of single components.

Production of reactive oxygen species (ROS) is believed to play a pivotal role in the etiology of PM-induced pulmonary irritation, leading to increased oxidative stress and subsequent inflammatory events locally and systemically. ${ }^{15} \mathrm{PM}$ from ambient air is known to be able to induce production of ROS in different cell types in vitro, ${ }^{16}$ but information about the oxidative capacity of indoor air particles is limited to studies on specific emission sources such as candles and incenses. ${ }^{17}$ In addition to

\section{Practical Implications}

- Oxidative capacity of settled dust is associated with moisture score and microbial components, but does not differentiate the moisture-damaged from the reference buildings.

- Hemolysis should be considered as one prospective mechanism contributing to the adverse health effects of moisture-damaged buildings.

combustion particles, microbial components can exert their effect via oxidative stress, ${ }^{18}$ indicating that PM collected from indoor environment may contain several components with high oxidative capacity.

Several of the microbial secondary metabolites isolated from fungi growing on building materials are potent toxins that can initiate immunomodulatory or cytotoxic effects. ${ }^{19}$ The prevalence and concentrations of individual toxins tend to be low even in moisturedamaged indoor environments, not differing much from reference buildings, ${ }^{20,21}$ unless extreme cases such as mold infestation after severe flooding are considered. However, the number of different metabolites detectable at elevated concentrations has been found to increase in moisture-damaged schools and residential buildings. ${ }^{20,21} \mathrm{~A}$ likely mode of action of mycotoxins in moisturedamaged indoor environments is to enhance the effect of other exposure agents rather than to cause acute toxicosis, as supported by studies on synergistic effects of co-exposure to mycotoxins and other biological agents. ${ }^{14}$ Direct evidence of adverse health effects of toxin exposure in moisture-damaged indoor environments at present is scarce, and mostly relating to sporadic cases of particularly serious moisture damage and the resulting microbial growth. However, studies of these cases have uncovered possible mechanisms of action for toxin-related respiratory distress, for example, hemorrhage in the lungs caused by metabolites of fungus Stachybotrys. ${ }^{22,23}$

The current study presents the oxidative capacity and hemolytic activity of a well-characterized set of settled dust samples from moisture-damaged and non-damaged schools from three European countries collected in the context of a pan-European epidemiological study. The results are correlated with the microbial content of samples and respiratory symptom prevalence among children in the sampled schools. 


\section{2 | MATERIALS AND METHODS}

\subsection{Study design and respiratory health assessment}

The schools participating in cross-sectional "Health Effects of Indoor Pollutants: Integrating microbial, toxicological and epidemiological approaches" (HITEA) project were selected and categorized as damaged or non-damaged as described earlier. ${ }^{24,25}$ In addition to the dichotomous categorization of each school, we utilized a moisture-damage score in the analysis (Appendix S1, p. 1). Settled dust samples were collected from 14 moisture-damaged (index) and 11 non-damaged (reference) schools in three countries: from Spain (five index + two reference schools), the Netherlands $(5+7)$, and Finland $(4+2)$. For the assessment of respiratory health, all children attending the schools were invited to complete a parent-administered survey based on the validated International Study of Asthma and Allergy in Children questionnaire. Respiratory symptoms (wheeze, congestion/phlegm, nocturnal dry cough, and rhinitis) and other symptoms possibly connected to hemolysis (nasal bleeding and fatigue) during the past 12 months were considered as the health outcome (Appendix S1, p. 2). The association between respiratory symptoms, school dampness and levels of microbial markers in these schools has been reported earlier by Jacobs et $\mathrm{al}^{26}$ and immunotoxicological potency of the studied settled dust samples by Huttunen et al. ${ }^{9}$

\subsection{Dust collection and extraction}

The settled dust samples were collected on cardboard boxes (settled dust boxes, SDBs) and electrostatic dustfall collectors (EDCs) ${ }^{27}$ placed at a height of 1.5-2.5 $\mathrm{m}$ for 8 weeks (Appendix S1, p.2). The processing of SDBs and EDCs has been described earlier. ${ }^{26}$ In brief, the dust accumulated in any given SDB box was vacuumed onto mixed cellulose ester (MCE) filters, suspended in dilution buffer, and stored frozen. A target of 15 settled dust box samples from different classrooms and other frequently occupied spaces in each school were pooled and aliquotted for subsequent analyses. The individual EDC cloths were extracted for endotoxin and glucan determinations as described earlier. ${ }^{28}$ The extract was centrifuged, aliquotted, and stored frozen in pyrogen-free glass tubes until analysis in central laboratory.

\subsection{Characterization of the dust sample}

The amount of selected microbial groups (fungal group Penicillium spp/Aspergillus spp/Paecilomyces variotii, the bacterial genus Mycobacterium spp, Gram-positive bacteria, Gram-negative bacteria) in the settled dust box samples were assessed with quantitative PCR assays as described earlier ${ }^{29-32}$ and chemical markers ergosterol and muramic acid by gas chromatography-tandem mass spectrometry (GC-MS-MS) with slightly modified methods published earlier. ${ }^{33,34}$ EDC extracts were analyzed for their endotoxin content with Limulus amoebocyte lysate ( $L A L)$ assay and glucans with a specific $\beta$-(1,3)-glucan sandwich enzyme immunoassay (EIA) as previously described by Noss et $\mathrm{al}^{35}$ (Appendix S1, p. 3). The particle count in settled dust box samples was measured with particle counter PAMAS SVSS (Appendix S1, p. 4). In addition, nine samples (two index and one reference schools per each country) were selected for detailed imaging with scanning electron microscopy (SEM) (Appendix S1, p. 5).

\section{4 | Plasmid scission assay}

Plasmid scission assay (PSA) was performed as described earlier by Koshy et al. ${ }^{36}$ The settled dust box samples were incubated with plasmid DNA and the relative amount of damaged (relaxed and linearized) DNA was assessed as percentage of all (including supercoiled intact) DNA (Appendix S1, p. 5). A dose-response curve was fitted on the data and the dilution capable of damaging $50 \%$ of the plasmid DNA (median toxic dilution, $\mathrm{TD}_{50}$ ) was determined to facilitate the comparison between samples. As the dependency is not linear, it was described with third-order polynomial fitted for each school separately. Similarly with the concept of median toxic dose, low median toxic dilution value indicates high bioactivity (Figure 1).

\section{5 | Hemolysis assay}

EDTA-anticoagulated blood was collected from two healthy human volunteers after informed consent and with approval of the local ethics committee. Hemolysis of isolated human red blood cells was measured after exposure to dust suspension (Appendix S1, p. 5). Absorbance of the supernatant was measured, and the percentage of hemolysis was calculated as the percentage of absorbance of each sample compared to the positive control. The average of the two tested donors was used to compare the samples with each other.

\subsection{Statistical analysis}

Basic tests and explorative analysis of the data were conducted with IBM SPSS Statistics 23.0 (IBM ${ }^{\circledR}$, New York, NY, USA). Since the variables are not normally distributed, differences between damaged

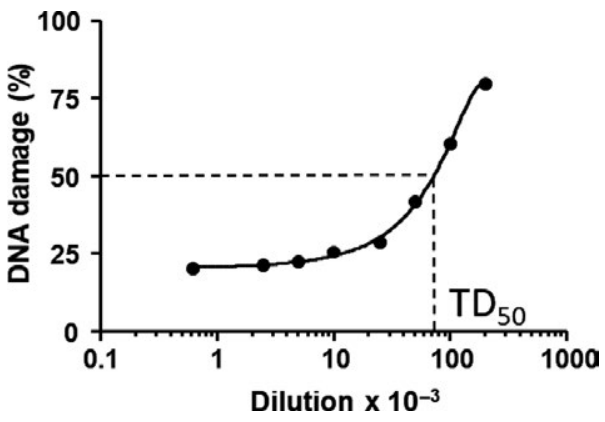

FIGURE 1 Determination of median toxic dilution $\left(T_{50}\right)$ value from third-order polynomial curve fitting to the plasmid scission assay (PSA) data. Example data from one Spanish index school 
and reference schools, countries as well as the comparison to carrier control were tested using non-parametric Mann-Whitney $U$ test or Kruskal-Wallis test. Associations between symptoms and hemolytic activity were evaluated using ORs with $95 \% \mathrm{Cl}$, obtained by multilevel mixed-effects logistic and linear regression. Associations with symptoms were adjusted for gender, age, parental educational level (as an indicator for socioeconomic level), and moisture damage in the home.

Structural equation modeling is a series of statistical methods that allow complex relationships between one or more independent variables and one or more dependent variables. ${ }^{37}$ A structural model represents hypotheses about effect priority. The model represents the hypothesis that $X$ is a cause of $Y$. Statistical estimates of direct effects are path coefficients, which are interpreted just as regression coefficients in linear model analysis. A special case of structural equation models, latent variable analysis (LVA) was deployed as the primary analytical technique to assess how the bacterial and fungal markers affect the $\mathrm{TD}_{50}$ values. Latent variables correspond to hypothetical constructs or factors, which are explanatory variables presumed to reflect a continuum that is not directly observable. For example here, we constructed latent variables corresponding to bacterial and fungal effect on the $\mathrm{TD}_{50}$ values, which are not directly observed but can be thought as weighted sums of individual markers. The number of observations did not allow for examining all the variables at the same time, as the model would have had too many estimable parameters compared to the data. However, we were able to construct separate models for fungal and bacterial components, both linked with oxidative capacity. Several criteria were used to evaluate the fit of the final model: for example, chi-square with degrees of freedom, the comparative fit index (CFI), and the root mean square error of approximation (RMSEA). These analyses were conducted using lavaan package ${ }^{38}$ in the R-software. ${ }^{39}$

\section{3 | RESULTS}

\subsection{Moisture score, particle count, and microbial components}

The moisture score was higher in index schools compared to reference schools, reaching statistical significance when the data from all three countries were combined. There were no statistically significant differences in the number of particles or amount of microbial components between the index and reference schools, even though the average amounts of microbial components such as Penicillium spp/Aspergillus spp/P. variotii, muramic acid, and Mycobacteria spp as well as Gram-positive and Gram-negative bacteria were generally higher in moisture-damaged environments (Table 1). The most prominent difference was seen comparing different countries; in most cases, the amount of the microbial components was highest in Spain and lowest in Finland (Figure 2).

TAB LE 1 Average $( \pm S E)$ moisture score, particle count, and amount of fungal and bacterial components in settled dust collected from Spain, the Netherlands, and Finland

\begin{tabular}{|c|c|c|c|c|c|c|c|c|}
\hline & \multicolumn{2}{|l|}{ Spain } & \multicolumn{2}{|c|}{ The Netherlands } & \multicolumn{2}{|l|}{ Finland } & \multicolumn{2}{|l|}{ All countries } \\
\hline Moisture score & $1.5 \pm 1.5$ & $4.8 \pm 0.6$ & $0.3 \pm 0.2$ & $2.4 \pm 0.5$ & $0.0 \pm 0.0$ & $4.0 \pm 0.4$ & $0.5 \pm 0.3^{*}$ & $3.7 \pm 0.4^{*}$ \\
\hline Particle count $\left(10^{3} / \mathrm{mL}\right)$ & $1100 \pm 300$ & $1300 \pm 400$ & $700 \pm 300$ & $600 \pm 100$ & $200 \pm 20$ & $200 \pm 60$ & $700 \pm 200$ & $700 \pm 200$ \\
\hline Glucan (ng/m² EDC) & $3900 \pm 400$ & $1700 \pm 100$ & $2800 \pm 600$ & $3500 \pm 500$ & $600 \pm 100$ & $800 \pm 200$ & $2600 \pm 500$ & $2100 \pm 400$ \\
\hline $\begin{array}{l}\text { Penicillium spp/Aspergillus } \\
\text { spp/Paecilomyces variotii } \\
\left(10^{3} \mathrm{CE} / \mathrm{SDB}\right)\end{array}$ & $40 \pm 5$ & $150 \pm 90$ & $20 \pm 10$ & $70 \pm 20$ & $10 \pm 10$ & $5 \pm 2$ & $20 \pm 10$ & $80 \pm 40$ \\
\hline \multicolumn{9}{|l|}{ Bacterial components } \\
\hline $\begin{array}{l}\text { Mycobacterium spp } \\
\left(10^{3} \mathrm{CE} / \mathrm{SDB}\right)\end{array}$ & $80 \pm 10$ & $150 \pm 60$ & $40 \pm 20$ & $70 \pm 20$ & $30 \pm 10$ & $30 \pm 20$ & $50 \pm 20$ & $80 \pm 20$ \\
\hline $\begin{array}{l}\text { Gram-negative bacteria } \\
\left(10^{3} \mathrm{CE} / \mathrm{SDB}\right)\end{array}$ & $1200 \pm 80$ & $4000 \pm 700$ & $400 \pm 200$ & $1300 \pm 600$ & $400 \pm 200$ & $400 \pm 200$ & $600 \pm 200$ & $2000 \pm 500$ \\
\hline $\begin{array}{l}\text { Endotoxin } \\
\left(10^{3} \mathrm{EU} / \mathrm{m}^{2} \mathrm{EDC}\right)\end{array}$ & $4.0 \pm 0.7$ & $5.0 \pm 1.8$ & $5.9 \pm 0.6$ & $11.6 \pm 1.8$ & $1.3 \pm 0.3$ & $1.3 \pm 0.2$ & $4.7 \pm 0.7$ & $6.30 \pm 1.5$ \\
\hline
\end{tabular}

CE, cell equivalent; EDC, electrostatic dustfall collector; EU, endotoxin unit; SDB, settled dust box; SE, standard error.

Statistically significant difference between index and reference schools is indicated with a star $\left({ }^{*} P<0.05\right.$, Mann-Whitney). 
Spain $\square$ The Netherlands $\square$ Finland

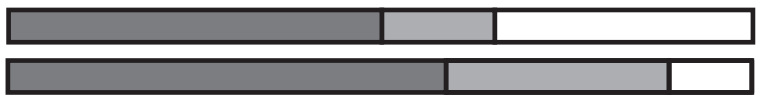

Moisture score*

Particle count ${ }^{*}$

\section{Fungal components}

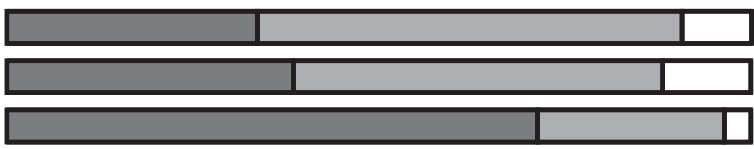

Ergosterol ${ }^{*}$

Glucan*

Penicillium/Aspergillus/

Bacterial components Paecilomyces variotii"

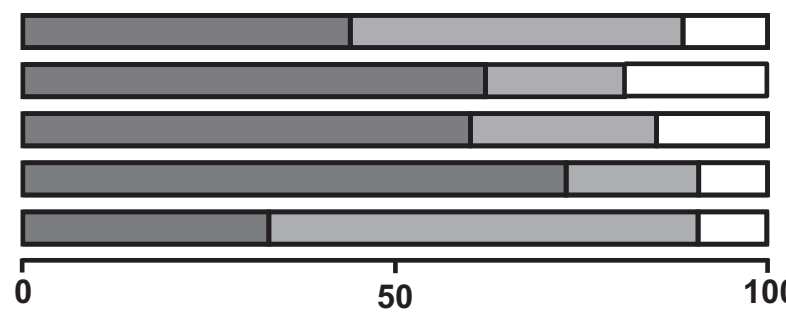

Muramic acid ${ }^{*}$

Gram-positive bacteria* Mycobacterium

Gram-negative bacteria* Endotoxin*

a statistically significant difference

( $P>0.05$, Kruskal-Wallis) between the countries.

\subsection{Scanning electron microscopy (SEM)}

The overall appearance of the samples showed accumulation of fine particulate matter with distinctive geological and biological origins in Spanish samples, heterogeneous material in smaller quantities in Dutch samples, and only few dispersed particulates in the Finnish samples (Figure 3). Overall, settled dust samples contained apparent biological structures such as spores, pollen, and hyphae (Figure 4A-E) as well as inorganic components such as mineral fragments and soot (Figure 4F). Samples from index and reference schools were not visibly different.

\section{3 | Oxidative capacity}

The oxidative capacity of the settled dust from Dutch schools was higher compared to Finnish and Spanish schools, but no statistically significant differences between the schools with and without moisture damages were found (Table 2, low $\mathrm{TD}_{50}$ values indicate high oxidative capacity).
To study the effects of bacterial and fungal markers on $\mathrm{TD}_{50}$, we constructed two LVA models with latent variables representing "bacterial factor" and "fungal factor." Amount of Gram-positive and Gram-negative bacteria, Mycobacteria spp and endotoxin in the sample were statistically significantly contributing variables in the construction of latent variable "bacterial factor" whereas the chemical markers ergosterol, glucan, and fungal group Penicillium spp/Aspergillus spp/P. variotii contributed significantly to the "fungal factor." In the first model, regressing $T D_{50}$ on the bacterial factor showed a notable negative effect, that is, increase in bacterial markers lead to lower $\mathrm{TD}_{50}$ and thus higher oxidative capacity of the sample. The number of particles was also inversely associated with $\mathrm{TD}_{50}$. The assessed moisture score of the schools had a direct increasing effect on the bacterial factor (Figure 5A). In the second model, both the fungal factor and the number of particles had a clear decreasing effect on the $T D_{50}$ value, but assessed moisture score of the schools affected the amount of the fungal group Penicillium spp/Aspergillus spp/P. variotii rather than the fungal factor directly (Figure 5B). In both models, the country where the samples were collected was a

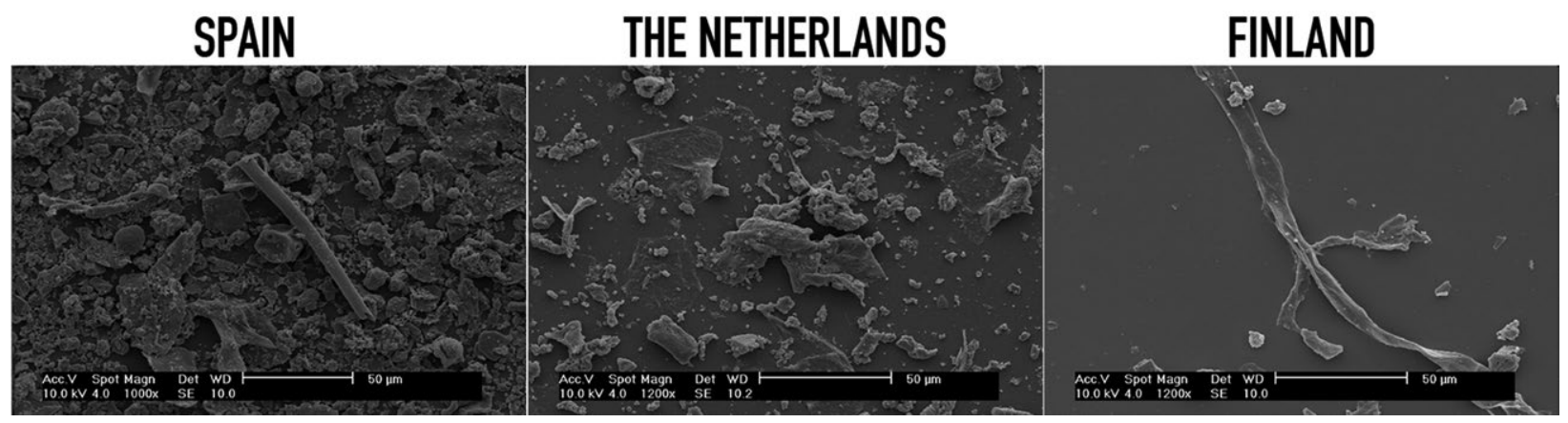

FIGURE 3 Scanning electron microscopy (SEM) pictures of settled dust samples from moisture-damaged schools in Spain (1000x), the Netherlands (1200x), and Finland (1200x) 

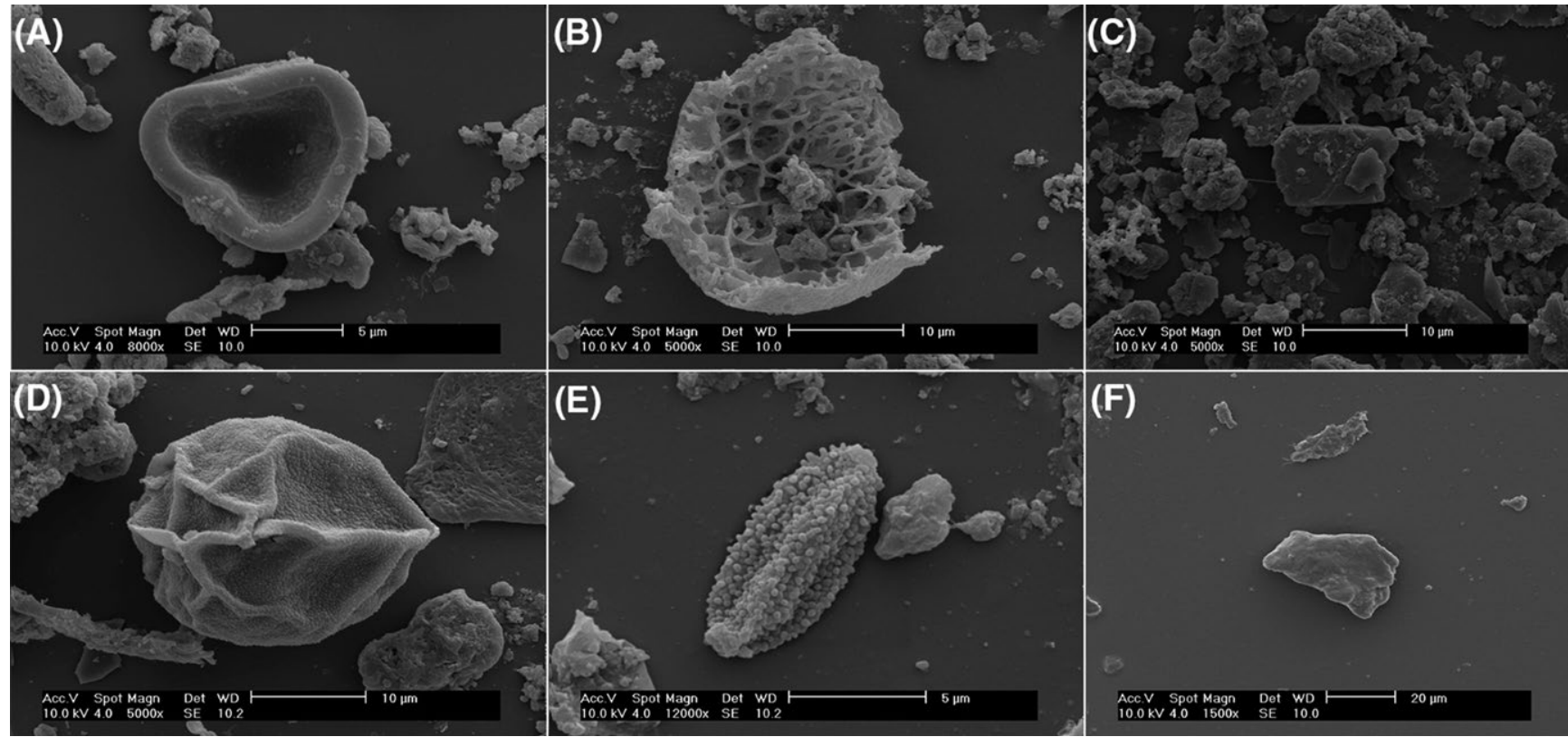

FIGURE 4 Scanning electron microscopy (SEM) pictures of biological and geological components featured in settled dust samples from schools in Spain (A-C), the Netherlands (D and E), and Finland (F)

significant factor affecting both $\mathrm{TD}_{50}$ and the bacterial/fungal factor along with moisture score and number of particles. According to fit indices, both LVA models were found to fit well to the data (eg, RMSEA < 0.1 and CFI > 0.95). Combinations of three or more microbial components significantly improved the fit of the model.

\subsection{Hemolytic activity}

Overall, the hemolytic activity of the dust samples was low but statistically significantly increased levels of hemolysis compared to control samples were seen in some of the moisture-damaged schools, specifically in dust from two out of five studied index schools from Spain (Figure 6). Differences between the index and reference schools or countries were not statistically significant, even though the elevated levels of hemolysis were detected only in moisture-damaged schools from one of the studied countries. Dry cough at night was reported more frequently in the schools with hemolytic samples both when comparing to Spanish reference schools (OR 1.8, 95\% Cl 1.61-2.01) or all the other Spanish schools (OR 1.49, 95\% Cl 1.19-1.87). Fatigue was reported less often in schools with hemolytic samples (OR 0.65, 95\% $\mathrm{Cl}$ 0.53-0.79), and no significant associations with wheeze, congestion/ phlegm, nasal symptoms, or nasal bleeding were seen.

\section{4 | DISCUSSION}

There are several possible mechanisms leading to adverse health effects after exposure to indoor air PM in moisture-damaged environments. We aimed to clarify if oxidative capacity or hemolytic activity of indoor air PM is associated with the moisture-damage status of the building or microbial components present in the sample material. While the oxidative capacity of the sample was linked with microbial composition of the sampled school, it did not differentiate the moisture-damaged schools from the non-damaged schools. Structural equation modeling showed that the oxidative capacity was influenced by grouped markers for bacteria (Gram-negative and Gram-positive bacteria, Mycobacterium spp and endotoxin) and fungi (ergosterol, $\beta$-glucan, and fungal group Penicillium spp/Aspergillus spp/P. variotii). Hemolytic activity was increased only in two out of 25 schools, but interestingly, these two Spanish buildings were both moisture-damaged schools and the children attending these schools reported nocturnal cough more often when compared to all other schools in this country; suggesting that on some occasions hemolytic activity could contribute to the adverse effects of indoor exposure.

Comparing the oxidative capacity of the settled dust to the microbial content of the sample revealed that both markers of bacterial

\begin{tabular}{|c|c|c|c|}
\hline Country & Reference schools & Index schools & All schools \\
\hline Spain & $64 \pm 33(2)$ & $55 \pm 7(5)$ & $58 \pm 9(7)$ \\
\hline The Netherlands & $33 \pm 6(7)$ & $27 \pm 5(5)^{*}$ & $31 \pm 4(12)^{*}$ \\
\hline Finland & $48 \pm 4(2)$ & $57 \pm 4(4)$ & $53 \pm 3(6)$ \\
\hline All countries & $41 \pm 7(11)$ & $46 \pm 5(14)$ & $44 \pm 4(25)$ \\
\hline
\end{tabular}

Statistically significant difference between countries is indicated with a star $\left({ }^{*} P<0.05\right.$, Kruskal-Wallis).
TABLE 2 Average ( \pm SE) oxidative capacity of settled dust from moisturedamaged (index) and non-damaged (reference) schools from Spain, the Netherlands, and Finland. Oxidative capacity is expressed as toxic dilution $\mathrm{TD}_{50} \times 10^{-3} \pm \mathrm{SE}(\mathrm{n})$, where lower number indicates higher oxidative capacity of the particulate matter 
FIGURE 5 Outcome of structural equation modeling showing standardized estimates of effects between latent variable "Bacterial factor" (A) or "Fungal factor" (B) and oxidative capacity (expressed as toxic dilution $\mathrm{TD}_{50}$, where low value indicates high oxidative capacity), moisture score of the school and number of particles in the sample. Coefficients are standardized linear regression weights, which can be used in examining the possible causal linkage between variables in the latent variable analysis (LVA) modeling approach. Standardized estimates follow normal distribution with mean of zero and variance of one. In addition to the variables in the figure, also country of origin had a significant effect on oxidative capacity, number of particles, moisture score, and both microbial factors
(A)

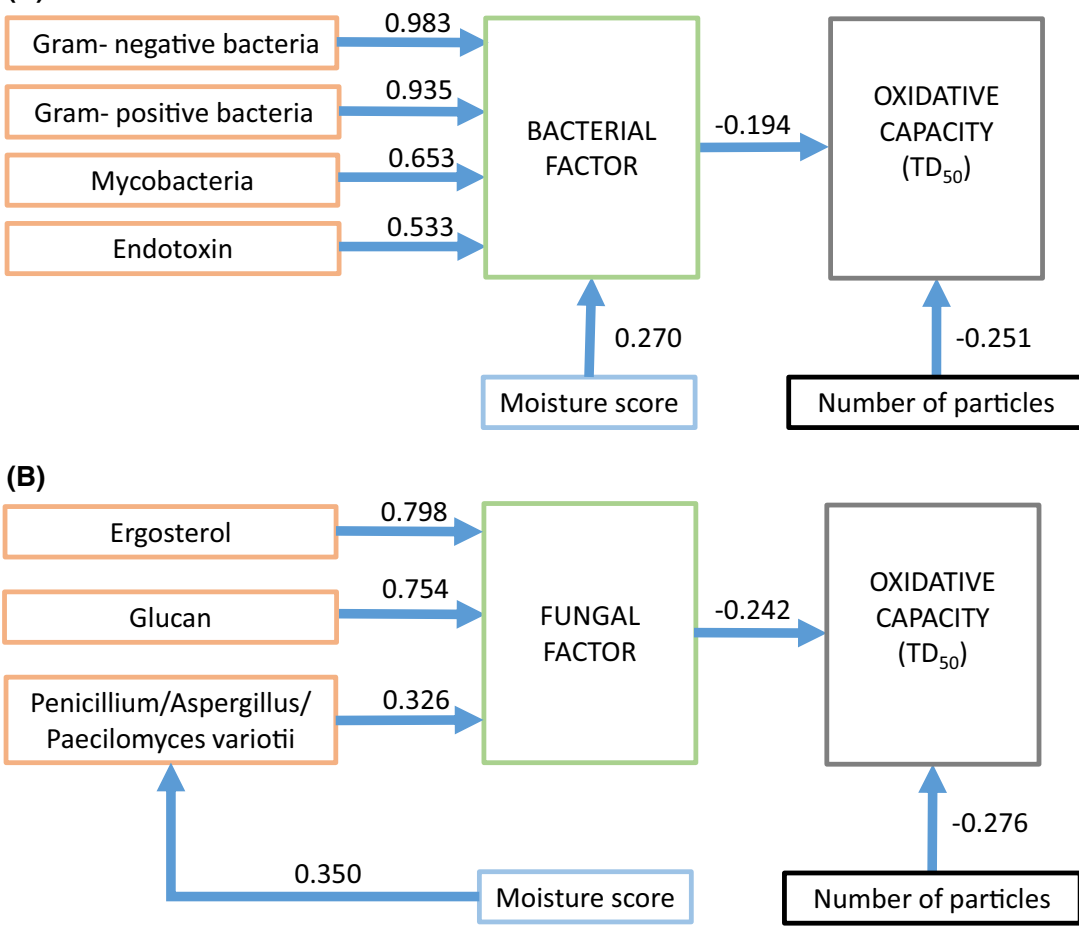

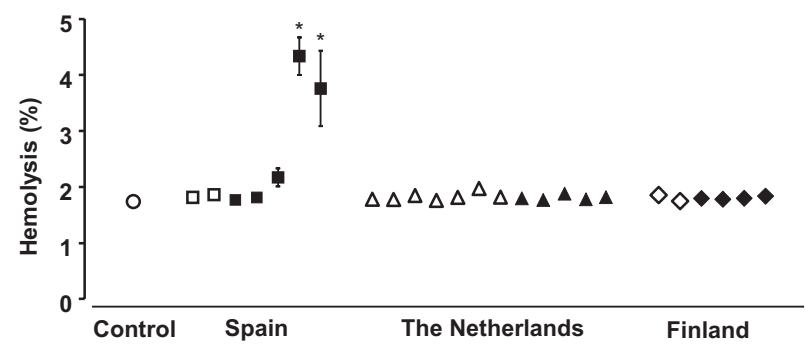

FIGURE 6 Average ( \pm SE) hemolysis after exposure to settled dust collected from 14 moisture-damaged (black symbols) and 11 non-damaged (white symbols) schools in Spain ( $\square$ ), the Netherlands $(\triangle)$, and Finland $(\diamond)$. Red blood cells from two donors were analyzed in triplicate $(\mathrm{N}=6)$. Statistically significant difference $(P<0.05$, Kruskal-Wallis) compared to control (Dulbecco's Phosphate Buffered Saline, PBS) is indicated with star $\left({ }^{*}\right)$

and fungal exposure were associated with increased oxidative stress. Importantly, combinations of three or more microbial components significantly improved the fit of the model, indicating that the presence of any single microbial component was not enough to explain the association. The association between the moisture damage and oxidative capacity is likely confounded by bacterial species or components being more strongly linked with other factors than moisture damage, such as occupancy, building factors, and outdoor levels. ${ }^{40}$ More refined classification of the buildings according to moisture observations showed that bacterial indicators as a group increased together with the moisture score, whereas the effect of moisture observations on fungi was reflected in the amount of the fungal group Penicillium spp/Aspergillus spp/P. variotii, that has been shown to be prevalent in moisture-damaged building materials. ${ }^{41}$ These results support our earlier findings on the immunotoxicological potential of the same samples, showing an association between in vitro responses and particularly bacterial components in the dust and strong effect of the country of origin, but no significant difference between schools classified as moisture-damaged and non-damaged. ${ }^{9}$ The low number of observations prevented us from including all variables in the same LVA model, but analyzing the data in two parts-separating bacterial from fungal factors-was successful. It would have been interesting to see the interactions also between the fungal and bacterial factors, but this remains to be explored in future studies with larger datasets. Unfortunately, we did not have the possibility to measure all possible factors affecting the oxidative capacity of the PM. For example, combustion exhaust particles have been previously linked with oxidative stress in several studies, ${ }^{42}$ and outdoor emissions are a major contributor to indoor PM. ${ }^{1}$

Hemolytic activity of indoor particulate sample is a noteworthy attribute for two reasons: firstly for the unlikely but possible direct adverse health effects of hemolysis such as pulmonary hemorrhage in exposed occupants and secondly for the indirect effect of increasing the concentration of free hemoglobin in the exposed tissues, leading to potent synergistic activation of innate immunity via Toll-like receptors. ${ }^{43}$ Hemolysins (ie, proteins and lipids causing lysis of RBC by destroying their cell membrane) were first recognized as the pathogenic product of Gram-positive bacteria even though several fungal species are able to produce hemolysins as well. ${ }^{44}$ In the context of indoor air microbial exposure, hemolytic activity has been associated with the fungal genus Stachybotrys. ${ }^{22,23}$ Due to the low number of hemolytic samples in our study, it was not possible to link the hemolytic activity with specific microbial components; for example, Stachybotrys was found to be present only in two samples 
which were not the ones causing hemolysis. However, the results indicate that settled dust from some of the studied moisture-damaged schools was hemolytic, and the activity was not confined to the samples positive for Stachybotrys species.

In conclusion, the association of oxidative capacity with the presence of microbial components suggests that in addition to inorganic elements, also organic fraction of the PM contributes to the oxidative stress caused by the exposure. The moisture score of the studied schools was associated with bacterial components as well as with a common group of fungal species in moisture-damaged buildings, but neither oxidative capacity nor hemolytic activity of the samples differentiated the moisture-damaged schools from non-damaged ones. Even though the indication of hemolytic activity of the settled dust was a rare find, hemolysis should be considered as one of the possible mechanisms contributing to the adverse health effects of moisture-damaged buildings.

\section{ACKNOWLEDGEMENTS}

We acknowledge the support of the European Respiratory Society (Fellowship LTRF 2013-1505), European Commission (Health Effects of Indoor Pollutants: Integrating microbial, toxicological and epidemiological approaches, HITEA, Grant agreement no. 211488 under the Seventh Framework Programme), The Helmholtz Virtual Institute of Complex Molecular Systems in Environmental Health (HICE), Nessling Foundation, The Academy of Finland Centre of Excellence (grant no. 272041), Olvi Foundation, Väinö and Laina Kivi Foundation, The Research Foundation of the Pulmonary Diseases, and The Cardiff Institute for Tissue Engineering \& Repair (CITER). ISGlobal is a member of the CERCA Programme, Generalitat de Catalunya.

\section{ORCID}

Kati Huttunen (iD https://orcid.org/0000-0002-4888-9203

\section{REFERENCES}

1. Jantunen M, Oliveira Fernandes E, Carrer P, Kephalopoulos S. Promoting actions for healthy indoor air (IAIAQ). Luxembourg, European Communities:European Commission Directorate General for Health and Consumers; 2011:5-16.

2. Wagner W, Sachrajda I, Pułaski L, Hałatek T, Dastych J. Application of cellular biosensors for analysis of bioactivity associated with airborne particulate matter. Toxicol In Vitro. 2011;25(5):1132-1142.

3. Happo M, Markkanen A, Markkanen P, et al. Seasonal variation in the toxicological properties of size-segregated indoor and outdoor air particulate matter. Toxicol In Vitro. 2013;27(5):1550-1561.

4. Long CM, Suh HH, Kobzik L, et al. A pilot investigation of the relative toxicity of indoor and outdoor fine particles: in vitro effects of endotoxin and other particulate properties. Environ Health Perspect. 2001;109(10):1019-1026.

5. Monn C, Becker S. Cytotoxicity and induction of proinflammatory cytokines from human monocytes exposed to fine (PM2.5) and coarse particles (PM10-2.5) in outdoor and indoor air. Toxicol Appl Pharmacol. 1999;155(3):245-252.
6. Roponen M, Meklin T, Rintala H, Hyvärinen A, Hirvonen MR Effect of moisture-damage intervention on the immunotoxic potential and microbial content of airborne particles and on occupants' upper airway inflammatory responses. Indoor Air. 2013;23(4):295-302.

7. Saraf A, Larsson L, Larsson BM, Larsson K, Palmberg L. House dust induces IL-6 and IL-8 response in A549 epithelial cells. Indoor Air. 1999;9(4):219-225.

8. Deschamps E, Weidler PG, Friedrich F, Weiss C, Diabaté S. Characterization of indoor dust from Brazil and evaluation of the cytotoxicity in A549 lung cells. Environ Geochem Health. 2014;36(2):225-233.

9. Huttunen K, Tirkkonen J, Täubel M, et al. Inflammatory potential in relation to the microbial content of settled dust samples collected from moisture-damaged and reference schools: results of HITEA study. Indoor Air. 2016;26(3):380-390.

10. Rylander R, Lin $\mathrm{RH}$. $(1 \rightarrow 3)$-beta-D-glucan: relationship to indoor air-related symptoms, allergy and asthma. Toxicology. 2000;152:47-52.

11. Park JH, Cox-Ganser JM, Kreiss K, White SK, Rao CY. Hydrophilic fungi and ergosterol associated with respiratory illness in a waterdamaged building. Environ Health Perspect. 2008;116:45-50.

12. Thorne PS, Mendy A, Metwali N, et al. Endotoxin exposure: predictors and prevalence of associated asthma outcomes in the United States. Am J Respir Crit Care Med. 2015;192(11):1287-1297.

13. Huttunen K, Pelkonen J, Nielsen KF, et al. Synergistic interaction in simultaneous exposure to Streptomyces californicus and Stachybotrys chartarum. Environ Health Perspect. 2004;112:659-665.

14. Korkalainen M, Täubel M, Naarala J, et al. Synergistic proinflammatory interactions of microbial toxins and structural components characteristic to moisture-damaged buildings. Indoor Air. 2017;27:13-23.

15. Kelly FJ, Fussell JC. Linking ambient particulate matter pollution effects with oxidative biology and immune responses. Ann N Y Acad Sci. 2015;1340:84-94.

16. Risom L, Møller P, Loft S. Oxidative stress-induced DNA damage by particulate air pollution. Mutat Res. 2005;592(1-2):119-137.

17. Chuang HC, Jones T, BéruBé K. Combustion particles emitted during church services: implications for human respiratory health. Environ Int. 2012;40:137-142.

18. Markkanen Penttinen P, Pelkonen J, Tapanainen M, et al. Co-cultivated damp building related microbes Streptomyces californicus and Stachybotrys chartarum induce immunotoxic and genotoxic responses via oxidative stress. Inhal Toxicol. 2009;21(10):857-867.

19. Jarvis BB, Miller JD. Mycotoxins as harmful indoor air contaminants. Appl Microbiol Biotechnol. 2005;66(4):367-372.

20. Peitzsch M, Sulyok M, Täubel M, et al. Microbial secondary metabolites in school buildings inspected for moisture damage in Finland, The Netherlands and Spain. J Environ Monit. 2012;14(8):2044-2053.

21. Kirjavainen PV, Täubel M, Karvonen AM, et al. Microbial secondary metabolites in homes in association with moisture damage and asthma. Indoor Air. 2016;26(3):448-456.

22. Vesper SJ, Vesper MJ. Stachylysin may be a cause of hemorrhaging in humans exposed to Stachybotrys chartarum. Infect Immun. 2002;70(4):2065-2069.

23. Yike I, Miller MJ, Sorenson WG, et al. Infant animal model of pulmonary mycotoxicosis induced by Stachybotrys chartarum. Mycopathologia. 2002;154(3):139-152.

24. Borràs-Santos $A$, Jacobs $\mathrm{JH}$, Täubel $M$, et al. Dampness and mould in schools and respiratory symptoms in children: the HITEA study. Occup Environ Med. 2013;70(10):681-687.

25. Haverinen-Shaughnessy $U$, Borras-Santos $A$, Turunen $M$, et al. Occurrence of moisture problems in schools in three countries from different climatic regions of Europe based on 
questionnaires and building inspections - the HITEA study. Indoor Air. 2012;22(6):457-466.

26. Jacobs J, Borràs-Santos A, Krop E, et al. Dampness, bacterial and fungal components in dust in primary schools and respiratory health in schoolchildren across Europe. Occup Environ Med. 2014;71(10):704-712.

27. Jacobs JH, Krop E, Spithoven JJ, de Wind S, Heederik D. Endotoxin levels in homes and classrooms of Dutch school children and respiratory health. Eur Respir J. 2013;42:314-322.

28. Noss I, Wouters IM, Visser M, et al. Evaluation of a low-cost electrostatic dust fall collector for indoor air endotoxin exposure assessment. Appl Environ Microbiol. 2008;74(18):5621-5627.

29. Haugland RA, Vesper SJ. Identification and quantification of specific fungi and bacteria. US Patent. 2002: 6387652.

30. Kaarakainen P, Rintala H, Vepsäläinen A, et al. Microbial content of house dust samples determined with qPCR. Sci Total Environ. 2009;407:4673-4680.

31. Torvinen E, Torkko P, Rintala AN. Real-time PCR detection of environmental mycobacteria in house dust. J Microbiol Methods. 2010;82:78-84.

32. Kärkkäinen PM, Valkonen M, Hyvärinen A, Nevalainen A, Rintala H. Determination of bacterial load in house dust using qPCR, chemical markers and culture. J Environ Monit. 2010;12:759-768.

33. Axelsson BO, Saraf A, Larsson L. Determination of ergosterol in organic dust by gas chromatography-mass spectrometry. J Chromatogr B. 1995;666:77-84.

34. Sebastian A, Larsson L. Characterisation of the microbial community in indoor environments: a chemical-analytical approach. Appl Environ Microbiol. 2003;69:3103-3109.

35. Noss I, Wouters IM, Bezemer G, et al. Beta-(1,3)-Glucan exposure assessment by passive airborne dust sampling and new sensitive immunoassays. Appl Environ Microbiol. 2010;76:1158-1167.

36. Koshy L, Jones T, BéruBé K. Bioreactivity of municipal solid waste landfill leachates -Hormesis and DNA damage. Water Res. 2008;42(8-9):2177-2183.

37. Hoyle R. Handbook of structural equation modeling. New York, NY: Guilford Press. (HSEM); 2012.
38. Rosseel Y. lavaan: An R package for structural equation modeling. J Stat Softw. 2012;48(2):1-36.

39. Core R, Team R. A language and environment for statistical computing. Vienna, Austria: R Foundation for Statistical Computing; 2017.

40. Rintala H, Pitkäranta M, Täubel M. Microbial communities associated with house dust. Adv Appl Microbiol. 2012;78:75-120.

41. Pietarinen VM, Rintala $H$, Hyvärinen $A$, et al. Quantitative PCR analysis of fungi and bacteria in building materials and comparison to culture-based analysis. J Environ Monit. 2008;10(5):655-663.

42. Øvrevik J, Refsnes M, Låg M, et al. Triggering mechanisms and inflammatory effects of combustion exhaust particles with implication for carcinogenesis. Basic Clin Pharmacol Toxicol. 2017;121(Suppl 3):55-62.

43. Cox KH, Cox ME, Woo-Rasberry V, Hasty DL. Pathways involved in the synergistic activation of macrophages by lipoteichoic acid and hemoglobin. PLoS ONE. 2012;7(10):e47333.

44. Nayak AP, Green BJ, Beezhold DH. Fungal hemolysins. Med Mycol. 2013;51(1):1-16.

\section{SUPPORTING INFORMATION}

Additional supporting information may be found online in the Supporting Information section at the end of the article.

How to cite this article: Huttunen K, Wlodarczyk AJ, Tirkkonen J, et al. Oxidative capacity and hemolytic activity of settled dust from moisture-damaged schools. Indoor Air. 2019;29:299-307. https://doi.org/10.1111/ina.12527 